\title{
State Diagnosis of Elevator Control Transformer over Vibration Signal Based on MEA-BP Neural Network
}

\author{
QingHui Song, ${ }^{1}$ QingJun Song, ${ }^{2}$ Linjing Xiao $\mathbb{D},{ }^{1}$ HaiYan Jiang $\mathbb{D}^{2},{ }^{2}$ and LiNa Li $\mathbb{D}^{2}$ \\ ${ }^{1}$ Department of Mechanical and Electronic Engineering, Shandong University of Science \& Technology, Qingdao 264005, China \\ ${ }^{2}$ Tai-an School, Shandong University of Science \& Technology, Tai-an 271009, China \\ Correspondence should be addressed to HaiYan Jiang; littlsunny@163.com
}

Received 25 September 2021; Revised 19 November 2021; Accepted 4 December 2021; Published 24 December 2021

Academic Editor: Zhaoye Qin

Copyright (C) 2021 QingHui Song et al. This is an open access article distributed under the Creative Commons Attribution License, which permits unrestricted use, distribution, and reproduction in any medium, provided the original work is properly cited.

Vibration analysis is considered as an effective and reliable nondestructive technique for monitoring the operation conditions of elevator control transformer. In the paper, a novel model using the Empirical Mode Decomposition (EMD), the empirical wavelet packet transform, the mind evolutionary algorithm (MEA), and the backpropagation (BP) neural network is proposed for elevator control transformer fault diagnosis. Firstly, the collected signal is smoothed by EMD, the intrinsic mode function (IMF) components with large noise are determined according to the correlation coefficient, the wavelet adaptive threshold denoising algorithm is used to process the noisy IMF components, and the IMF components before and after processing and its residual component are reconstructed to obtain the denoised signal. Then, the denoised signal is transformed by empirical wavelet packet transform to extract the energy ratio and energy entropy features in the wavelet packet coefficients. Finally, a fault diagnosis model composed of MEA and BP neural network is developed, which avoids the problems of premature convergence and poor diagnosis effect. The experimental results show that the proposed model has a remarkable performance with an average root mean square error of 0.00672 and the average diagnosis accuracy of $90.8 \%$, which is better than classic BP neural network.

\section{Introduction}

The control transformer depresses the power voltage to provide the appropriate working voltage for the control loop. It is of great significance to study the fault diagnosis method for the transformer in the stable and safe operation of power control system $[1,2]$. So, many researchers have devoted themselves to the research of its diagnostic methods in recent years.

Up to now, many monitoring techniques and detection methods about transformer fault have been proposed. $\mathrm{Li}$ et al. introduced a power transformer fault diagnosis method based on an optimized generalized regression neural network by integrating with characteristic gas, cuckoo search algorithm, and rough set theory. Experimental results indicated that the proposed method could provide a useful solution for power transformer fault diagnosis [3]. Yang et al. presented a power transformer fault diagnosis approach using the dissolved gas analysis, combining the probabilistic neural network with the bat algorithm to improve the accuracy of fault diagnosis [4]. Meanwhile, they made an oil-immersed transformer fault diagnosis approach by a hybrid machine learning method which was constructed by Probability Neural Network by integrating with characteristic gas and Multiverse Optimizer algorithm [5]. In [6], the imprecise probability theory was used to evaluate the properties of each type of transformer fault by the naive credal classifier and the imprecise Dirichlet estimation model. Hoballah et al. developed a power transformer fault diagnosis method based on dissolved gas percentages, and the fuzzy system and hybrid grey wolf (HGW) optimization algorithm were utilized to produce the fault types and the diagnostic code matrix. Genetic algorithm and Particle Swarm Optimization were added to the HGW optimizer to obtain the global optimal solution [7].

In the transformer fault diagnosis of dissolved gas analysis, the accuracy of fault diagnosis is greatly affected by the uncertainty of measurement data in the process of oil 
sample collection, processing, and storage [7]. Therefore, methods based on characteristic gas are not always efficient in some cases [8]. Fault diagnosis of transformer by vibration approach is a new area [9]; therefore, in decades, people have been trying to utilize the transformer vibration signal to analyze the transformer faults [10].

Bartoletti et al. introduced a transformer diagnostic procedures for medium voltage/low voltage transformers based on vibration and acoustic methods, which could distinguish new, used, and anomalous transformers [11]. Hong et al. introduced a winding condition assessment method in the light of the fundamental frequency vibration analysis of vibration variations. Then, the correlation of windings vibration of different sensors was analyzed by principal component analysis theory, and two health parameters were proposed in the model, which, respectively, represented the degree of vibration correlation and the fault location [9]. Zhou et al. developed a winding vibration model by electromagnetic force analysis and analyzed the winding vibrations. Numerical simulation results show that the vibration is in line with the expected value when the clamping force is large enough [12]. Liu et al. analyzed the main cause of vibration and noise of transformer body and put forward a transformer vibration transmission model to analyze the vibration relationship between transformer core and shell [13]. Zhang et al. analyzed the coupling and vibration transmission attribute of winding vibration and core vibration for single-phase power transformer. And the experimental results show that the coupling response of winding vibration and core vibration has a broader frequency band than that caused by electromagnetic force [14]. Cao et al. proposed a method of monitoring the looseness state and deformation fault of transformer windings based on vibration and reactance information. The complete ensemble EMD transform method was used to extract the vibration characteristic information, and the vibration relationship between core and shell was deduced theoretically [15]. The vibration of the extra-high-voltage transformer tank was measured, and the load current, voltage, and oil temperature were investigated. It was pointed out that the natural vibration frequency of the winding is a more suitable diagnostic index [16]. Hong et al. analyzed the influence of load current on winding vibration response and proposed a transformer condition monitoring method based on gated recurrent unit neural network. The result showed that the electromagnetic force caused by the load current will affect the vibration response of the winding structure [17]. In [18], Amin et al. investigated the possibility of using deep neural networks to capture the hidden modes of vibration time series to predict the progress of transformer faults under early excitation and interturn. And the vibration analysis is considered to be an efficient and nondestructive technique for monitoring transformer operating conditions. In [19], a condition monitoring method of transformer for vehicular was proposed, and the geographical location, driving speed, vibration, and impact acceleration information of the onboard mobile transformer were obtained in real time. Zhang et al. proposed a transformer fault diagnosis method based on Internet of Things and ensemble machine learning.
Firstly, deep belief networks and stacked denoising autoencoders were used to extract features from vibration signals, respectively. Then, a new ensemble machine learning method based on deep belief networks and relevance vector machines was constructed to carry out transformer fault diagnosis experiments [20]. Hong et al. introduced a novel feature extraction method based on vibration analysis by transforming vibration monitoring information into feature images. Then, a condition monitoring approach was put forward in combination with deep learning and convolutional neural network to classify the winding conditions, including normal, degraded, and anomalous [21]. Xiao et al. proposed a multiscale fusion feature extraction model for transformer based on the measured vibration data. Firstly, the vibration signals were converted into the time domain, frequency domain, and energy characteristic images by using Markov Transform Field and Continuous Wavelet Transform, and then multiparallel convolutional neural network was introduced to realize the fault detection and identification of transformer [22].

At present, the vibration signal analysis method and the artificial intelligence technology are recognized as one of the effective methods of power transformer fault detection. However, there is little research on online monitoring of elevator control transformer. In this paper, a new method of transformer fault diagnosis based on empirical wavelet packet, mind evolutionary optimization algorithm, and BP neural network is proposed. First, we calculate the IMF of vibration signals and the correlation between each IMF and the original signal and carry out wavelet adaptive threshold denoising for the components with low correlation. Then, an improved wavelet packet transform is proposed to decompose the vibration signal more precisely. At the same time, the energy ratio and energy entropy of empirical wavelet packet are utilized to construct the feature set of control transformer fault diagnosis. Using the improved neural network MEA-BP, combined with the constructed features, the operation state detection and fault diagnosis of the control transformer are realized.

\section{Monitoring Device and Method}

2.1. Monitoring Device. Vibration analysis is an economical and effective nondestructive technique for monitoring transformer operation $[18,23]$. In the work, a condition monitoring system of elevator control transformer on the basis of vibration signal analysis is designed, which includes current sensor, voltage sensor, vibration sensor, temperature sensor, data acquisition card, and computer. The system schematic diagram is shown in Figure 1.

In this study, the data acquisition card PXI449 is selected which was specially designed for vibration by Ni Company, and the application program of the state diagnosis system of elevator control transformer was designed by using LabView software.

Installation positions of vibration sensor and temperature sensor are shown in Figure 2. The vibration sensor is adsorbed on the transformer shell, which can detect the vibration signals sent by components such as transformers 


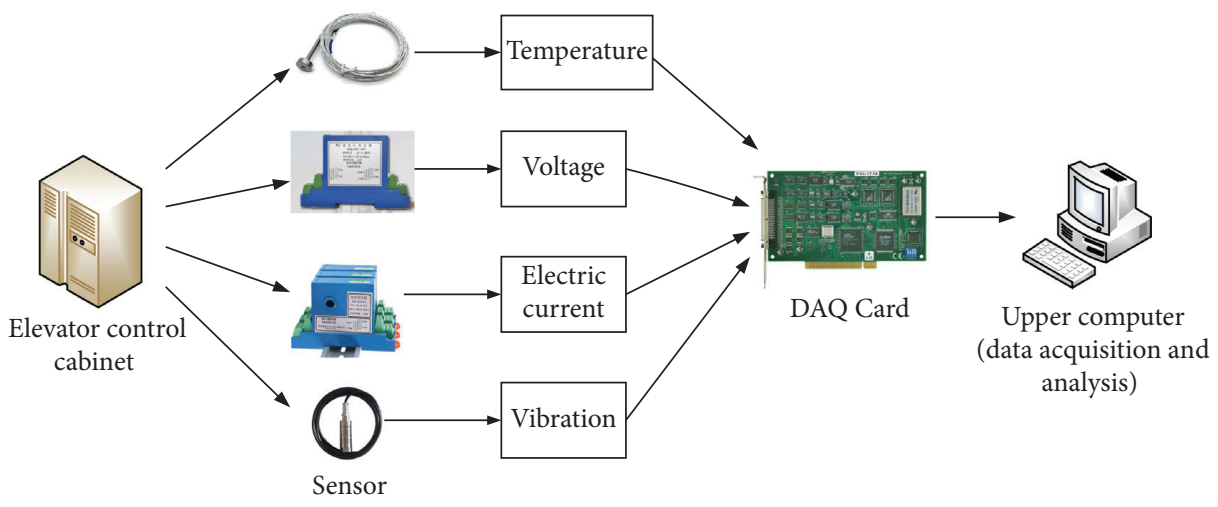

FIgURE 1: Schematic diagram of the condition monitoring system of elevator control transformer.

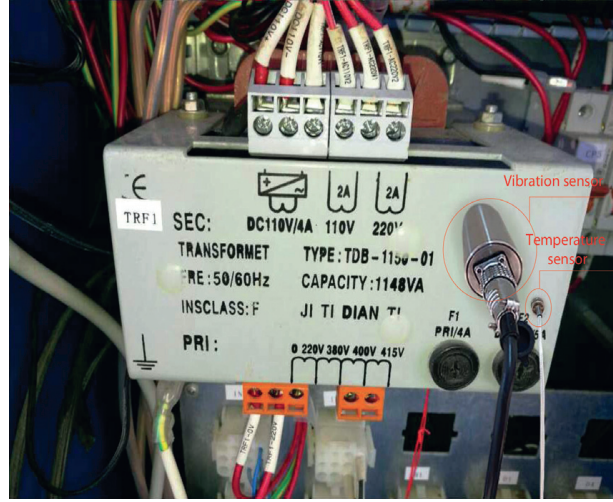

FIGURE 2: Vibration sensor and temperature sensor installation point.

and relays. The temperature sensor is adsorbed on the transformer housing to measure the transformer temperature.

The installation measuring points of voltage transmitter and current transmitter are shown in Figure 3. Two sensors are mounted on the mounting rail of the elevator control transformer. The voltage transmitter is connected to the direct-current (DC) bus of the frequency converter, and the input voltage of the frequency converter is collected. Current transmitter uses Hall induction principle to collect the output current of frequency converter.

In this work, the research object was Hitachi YP-15CO90 alternating current (AC) speed adjustable passenger elevator control cabinet, with a rated load of 15 people. The frequency range of vibration signal generated during transformer operation is $10-2000 \mathrm{~Hz}$. Combined with the characteristics of vibration signal on the surface of transformer box, CT1010LC vibration sensor is selected. The frequency response of the sensor is stable in the working bandwidth range of $50-10000 \mathrm{~Hz}$, which can provide accurate data for transformer fault diagnosis later. In this work, 500 cases of transformer operation state in YP-15CO90 were collected with a sampling frequency of $7 \mathrm{kHz}$, mainly including normal operation, winding fault, and core fault. 370 groups of cases were randomly selected as the training data of the fault diagnosis model, and the remaining

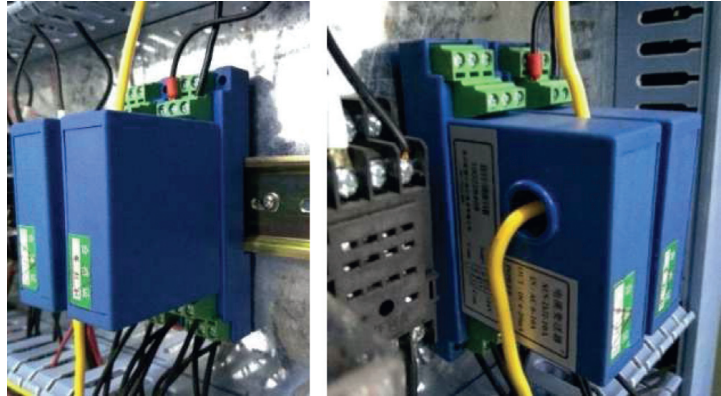

FIGURE 3: Voltage transmitter and current transmitter installation point.

130 groups of cases were used as the test data. The effectiveness of the proposed transformer fault diagnosis model is evaluated by 10 times cross validation.

The core fault signal waveform diagram is shown in Figure 4. As can be seen from the figure, when the elevator control transformer fails, the fluctuation of its vibration signal is irregular and no fault information can be extracted from it. Therefore, it is necessary to process the vibration signal to obtain the characteristics that can reflect the fault of elevator control transformer. This part will be discussed in detail in Section 3.

2.2. Method. The vibration of the transformer in the elevator control cabinet is mainly caused by the vibration of the transformer body structure, and its propagation path is shown in Figure 5.

According to the transformer function, transformer fault can be divided into subsystem faults such as iron core, winding, cooling, and mechanical structure. The deformation of iron core and winding in transformer is one of the important causes of transformer fault, which can be reflected by transformer surface vibration signal. The vibration of the coil winding is caused by the interacting electromagnetic force after the current flows through the coil. During the operation of the transformer, it may be impacted by the short-circuit current, and each coil may be affected by the strong radial force and axial force. In this case, the winding may deform axially and radially at the same time. The loose 


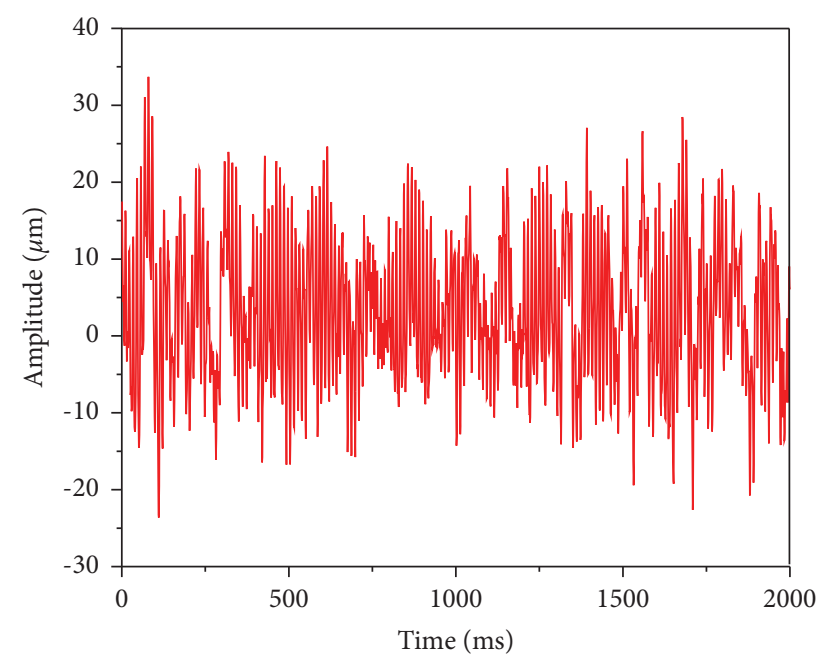

FIgURE 4: Vibration time domain diagram of core fault signal.

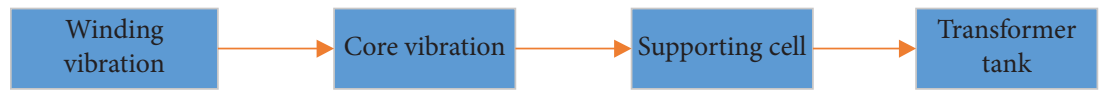

FIGURE 5: Vibration propagation path of the elevator control transformer.

deformation of winding will aggravate the imbalance of electromagnetic axial force, which will aggravate the vibration. Because the compressive stress of the core is different between normal and loose and the magnitude of the compressive stress will change the permeability and magnetostriction of the core, it will change the core vibration. The vibration frequency of the iron core mainly focuses on the integral multiple frequency of $50 \mathrm{~Hz}$ and also contains high-order harmonic components such as $200 \mathrm{~Hz}, 300 \mathrm{~Hz}$, and $400 \mathrm{~Hz}$. The winding vibration signal is mainly concentrated at the position of $100 \mathrm{~Hz}$ fundamental frequency component.

Figure 6 shows the amplitude of vibration signals in three states of transformer at the test position in Figure 2 at the frequency of $100-1000 \mathrm{~Hz}$. Obviously when the transformer is in normal operation, except for the $100 \mathrm{~Hz}$ vibration signal, the amplitude of each frequency changes very little within $1000 \mathrm{~Hz}$. When the core failure occurs, the amplitude of each frequency of the vibration signal changes greatly within $1000 \mathrm{~Hz}$. However, when the winding failure occurs, the amplitude of the high harmonic frequency fluctuates little less except that the amplitude of $100 \mathrm{~Hz}$ component increases greatly. Therefore, the characteristic quantity reflecting the fault information can be obtained by measuring and analyzing the vibration signals on the surface of the transformer box.

Aiming at the problem that it is difficult to distinguish between the faults of elevator control transformer components and the faults under the influence of high-order harmonics, the vibration method is considered to diagnose transformer. Transformer vibration signals are nonstationary, and the feature obtained only by empirical wavelet packet transform cannot directly reflect the transformer operation state, resulting in poor classification ability and low recognition accuracy of

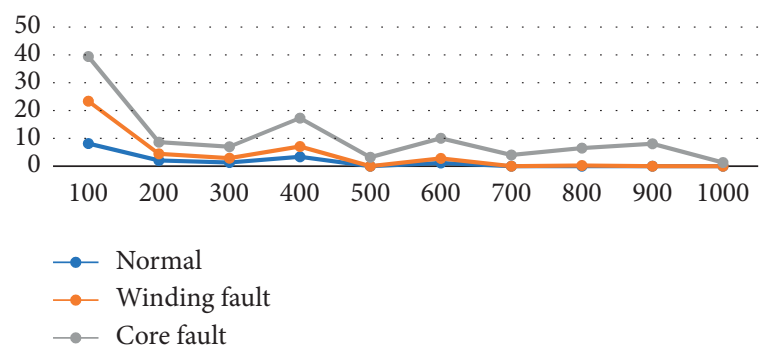

FIgURE 6: Comparison of frequency amplitude before and after failure.

signals. In this paper, EMD is used to stabilize the vibration signal and obtain a series of intrinsic mode functions. Secondly, the denoised signal is obtained using the correlation coefficient between the IMF component and the original signal, combined with wavelet threshold denoising method to reconstruct the signal. Then, the empirical wavelet packet technology is used to extract the energy ratio and energy entropy of some frequency bands to form robust signal features. Finally, the weight and threshold of neural network are optimized by mind evolutionary algorithm, and the optimized neural network model is used to diagnose transformer fault. The flowchart of the proposed method is illustrated in Figure 7.

\section{Theory}

When analyzing nonstationary transformer vibration signals, it is easy to cause low accuracy of transformer fault identification by EMD and wavelet packet transform (WPT) alone. This paper presents a feature extraction method of transformer vibration signal based on EMD and empirical wavelet packet (EWP) transform. 


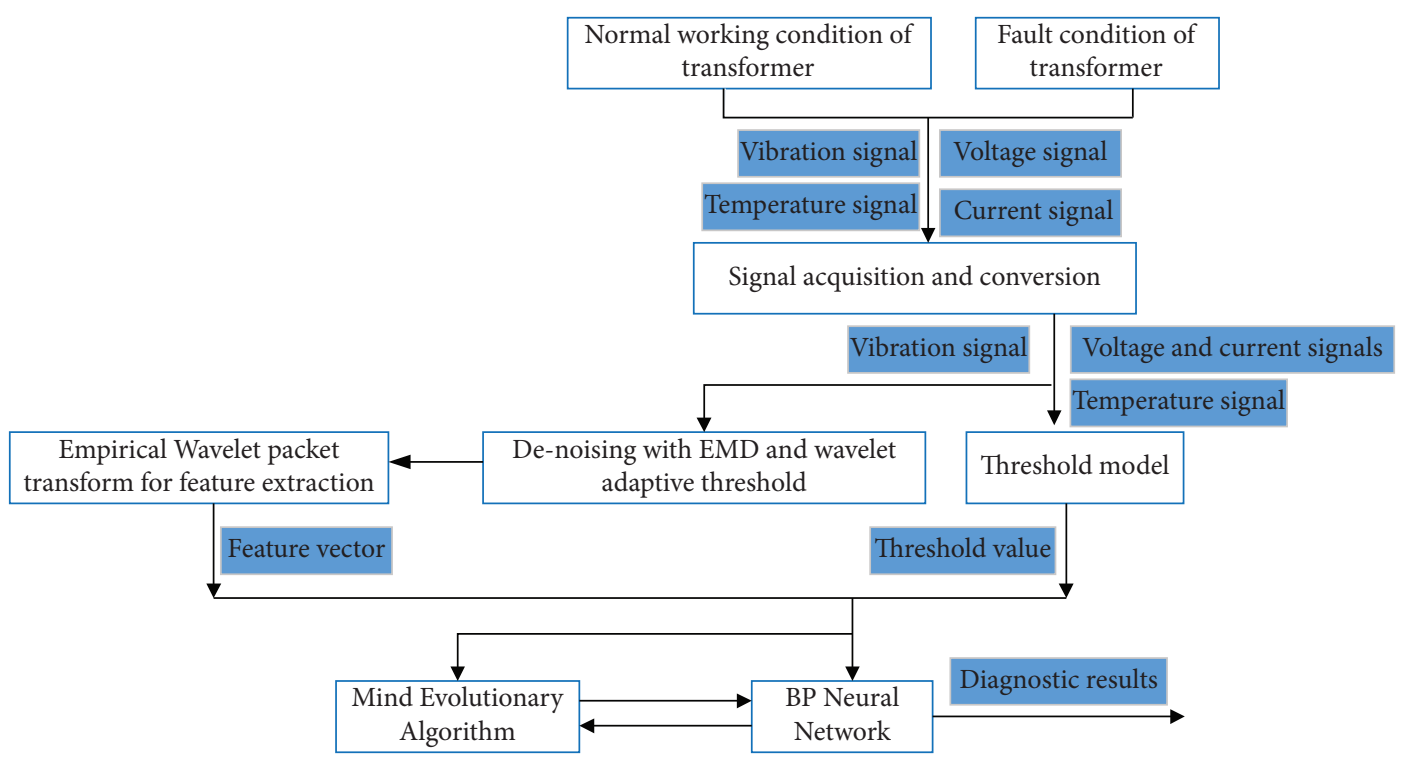

Figure 7: Flowchart of the proposed diagnostic scheme.

3.1. Denoising Algorithm Based on EMD and Wavelet Adaptive Threshold. The IMF components decomposed by EMD algorithm may produce redundant components due to overdecomposition. Therefore, it is necessary to select the most effective component. Simply selecting several orders of IMFs to extract features cannot achieve good recognition effect, and the correlation between IMF decomposed by different operating states of transformer and original signal is different. Firstly, EMD algorithm is used to decompose the transformer vibration signal, and the correlation coefficient between each IMF component and the original vibration signal is obtained. The IMF component with large noise is determined according to the arrangement of correlation coefficient, and the noise in the IMF component is removed by an adaptive wavelet threshold denoising method. Then, the IMF components before and after processing and their residual components are superimposed according to the ranking of correlation coefficients, and the denoised signal can be obtained after reconstruction. The simplified flow illustrating this method can be seen in Figure 8. Here, $\gamma$ is a weight parameter.

Assuming that the collected vibration signal of transformer operation is discrete as $x(n)_{n=1,2, \ldots, N}$, it is decomposed into different IMF function components by EMD method, and the expression is

$$
x(n)=\sum_{i=1}^{k} c_{i}(n)+r_{k}(n),
$$

where $r_{k}(n)$ is the residual quantity, representing the average trend term of the signal, and $c_{i}(n)$ is the $i$-th intrinsic mode functions component.

The correlation coefficient $C_{r}$ between all IMF components and the original signal can be expressed as

$$
C_{r}=\frac{\sum_{i=0}^{N}\left(x_{i}-\bar{x}\right)\left(p_{i}-\bar{p}\right)}{\sqrt{\sum_{i=0}^{N}\left(x_{i}-\bar{x}\right)^{2} \sum_{i=0}^{N}\left(p_{i}-\bar{p}\right)^{2}}},
$$

where $x_{i}$ is the original signal data point, $p_{i}$ is IMF component data point, and $\bar{x}$ and $\bar{p}$ are the average values of signals corresponding to $x_{i}$ and $p_{i}$, respectively.

Firstly, 100 groups of vibration data of elevator control transformer are randomly selected and EMD decomposition is carried out according to the correlation with the original signal. Then, correlation coefficients are calculated according to equation (2) and their average values are shown in Figure 9. It is not difficult to find that the correlation between the first four IMFs and the original signal is larger, indicating that the main information of the signal is contained in the first four IMF components. Considering the complexity and accuracy of the algorithm, $\gamma$ is set to 0.15 according to Figure 9 .

In wavelet threshold denoising, threshold plays an important role in distinguishing useful signal and noise. Here, an adaptive wavelet threshold function [24] is adopted as

$\bar{w}_{j, k}=\left\{\begin{array}{ll}\operatorname{sign}\left(w_{j, k}\right)\left(\left|w_{j, k}\right|-\frac{\lambda}{\left(w_{j, k}^{2}-\lambda^{2}\right)^{J}+1}\right), & \left|w_{j, k}\right| \geq \lambda \\ 0, & \left|w_{j, k}\right|<\lambda\end{array}\right.$,

where $\lambda$ and $j$ are the threshold value and wavelet decomposition layer, respectively. $w_{j, k}$ and $\bar{w}_{j, k}$ are the wavelet coefficient and quantized wavelet coefficient, respectively.

Obviously, when $w_{j, k}= \pm \lambda$, the quantized wavelet coefficient $\bar{w}_{j, k}=0$; when $w_{j, k}$ is close to $y$, the curve $\bar{w}_{j, k}=w_{j, k}$ is the asymptote of the improved threshold function. It can be seen that the new threshold function has continuity and is asymptotic, which overcomes the defects of pseudo Gibbs phenomenon in hard threshold function and invariant deviation of soft threshold function.

In order to compare the noise reduction effects with different wavelet basis functions, the peak signal-to-noise 


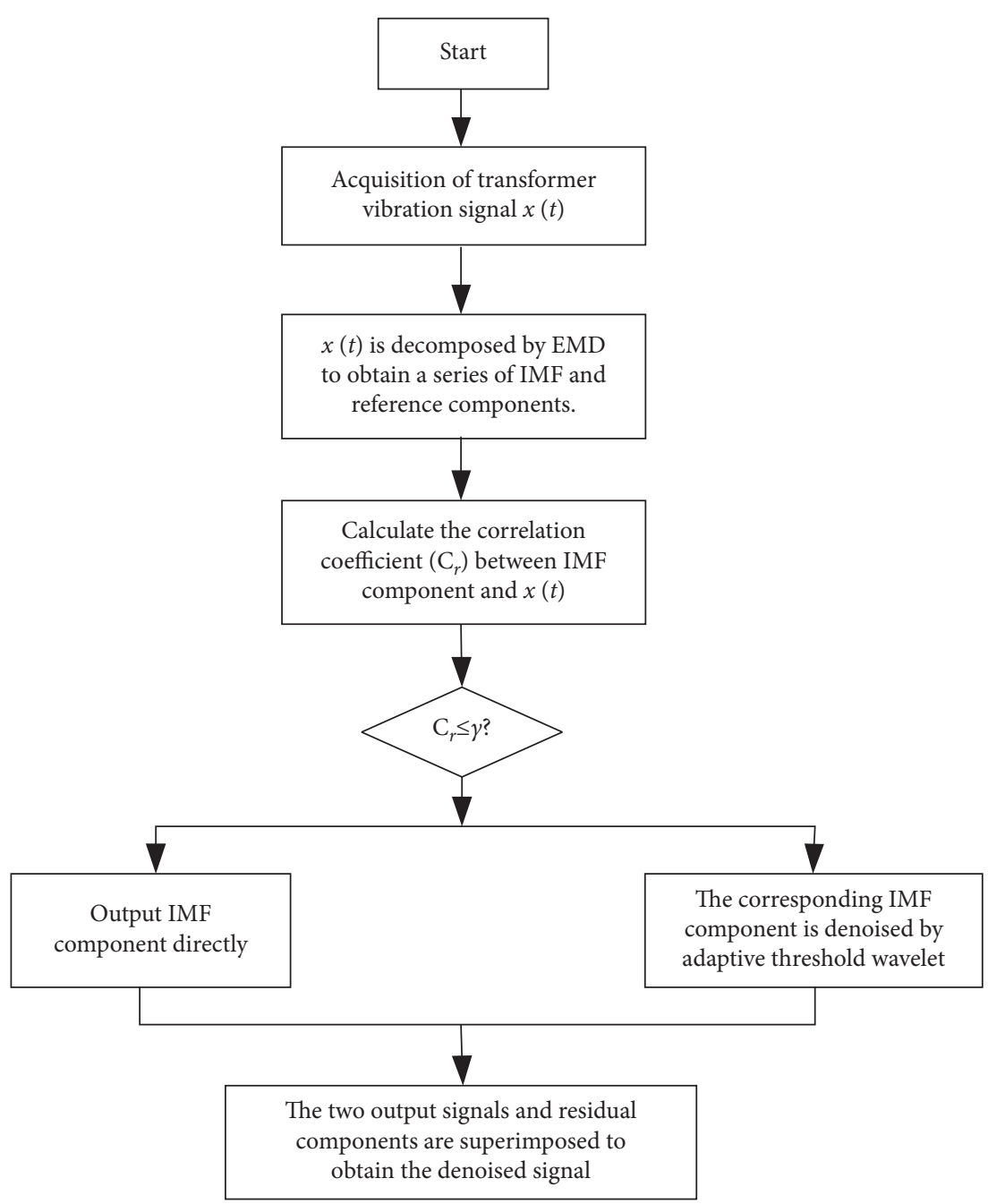

FIGURE 8: Flowchart of improved EMD and wavelet adaptive threshold denoising algorithm.

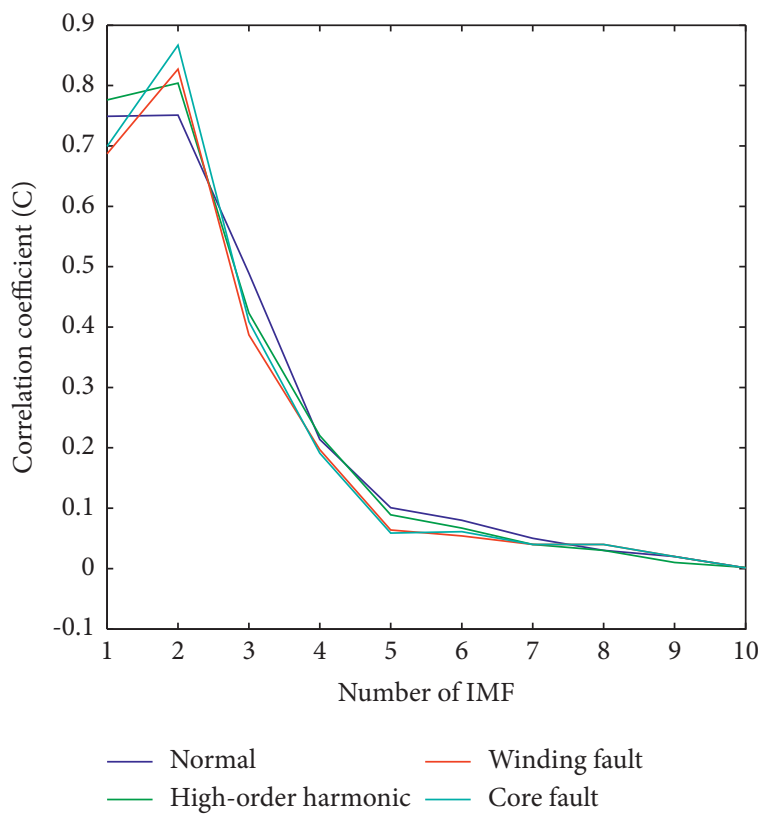

FIgURE 9: Average value of correlation coefficient. ratio (PSNR) is introduced as the objective evaluation standard. Table 1 shows the denoising performance of different wavelet basis functions. It can be found that sym6 had better results in denoising than the other families analyzed, namely, Daubechies and Biorthogonal. Here, based on sym6 wavelet, the noise component is denoised adaptively according to equation (3). According to the adaptive wavelet threshold denoising algorithm and the VisuShrink threshold method, SureShrink threshold method, and BayesShrink threshold method, the collected vibration signals are denoised, and the PSNRs are 24.5392, 23.4508, 23.7209, and 23.9531, respectively. The adaptive wavelet threshold denoising algorithm is obviously better than the traditional threshold function in denoising noisy signals.

Therefore, the improved EMD and wavelet adaptive threshold denoising algorithm can improve the accuracy of signal reconstruction and obtain relatively good denoising effect.

3.2. Empirical Wavelet Packet Transform. In wavelet decomposition, the time resolution of high-frequency band signal is high and the frequency resolution is low, and the 
TABLE 1: Denoising performance of different wavelet functions.

\begin{tabular}{lccccc}
\hline & $\mathrm{db} N$ & & sym $N$ & & biorNr.Nd \\
$\mathrm{dbN}$ & PSNR $(\mathrm{dB})$ & sym $N$ & PSNR (dB) & biorNr.Nd & bSNR $(\mathrm{dB})$ \\
\hline Db1 & 21.1756 & sym1 & 21.2425 & bior1.1 & 21.1033 \\
Db2 & 22.1503 & sym2 & 21.8625 & bior1.5 & 21.4539 \\
Db3 & 22.6234 & sym3 & 22.0526 & bior2.2 & 21.3648 \\
Db4 & 22.7801 & sym4 & 22.9627 & bior2.4 & 22.9247 \\
Db5 & 22.6018 & sym5 & 23.6429 & bior3.3 & 23.1184 \\
Db6 & 22.4085 & sym6 & 24.5392 & bior4.4 & 24.0186 \\
Db7 & 22.0049 & sym7 & 24.3201 & Bior5.5 & 23.8254 \\
Db8 & 21.7803 & sym8 & 23.9827 & & 22.1622 \\
\hline
\end{tabular}

time resolution of low-frequency band signal is low and the frequency resolution is high. Wavelet packet transform, also known as the optimal subband tree structuring, is a further optimization of wavelet transform, which decomposes the parent waveform not only into the low-frequency subband, but also into the high-frequency subband [25], thus improving the frequency resolution. Although wavelet packet transform is widely used in practical applications, it is still a designated wavelet transform rather than an adaptive transform of wavelet family. Gilles [26] proposed a novel signal decomposition method named empirical wavelet transform; its main idea is to design a suitable wavelet filter bank to extract the different modes of the amplitude modulation and frequency modulation of the original signal. Based on the wavelet packet theory, this paper introduces the scale function and wavelet function of empirical wavelet constructed by empirical wavelet transform technology to realize the adaptive transformation of wavelet family.

Suppose the angular frequency of the original signal is $\omega$ $(\omega \in[0, \pi])$, and divide the signal $[0, \pi]$ into $N$ intervals, and let the bandwidths of these intervals be different; then the $N$ th interval can be expressed as

$$
\begin{aligned}
\Lambda_{n} & =\left[\omega_{n-1}, \omega_{n}\right], \quad n=1,2, \ldots, N \\
\bigcup_{n-1}^{N} \Lambda_{n} & =[0, \pi] .
\end{aligned}
$$

Taking each angular frequency $\omega_{\mathrm{n}}$ as the center point, define the bandwidth interval of a transition section, and the width of the transition section is $T_{n}=2 \tau_{n}$ (see Figure 10).

Therefore, a tight framework can be implemented in the following form [27]:

$$
\tau_{n}=\gamma \omega_{n},
$$

where $\gamma$ is a frequency scale factor in the range $(0$, $\left.\min _{n}\left(\omega_{n+1}-\omega_{n} / \omega_{n+1}+\omega_{n}\right)^{8}\right)$.

After confirming interval $\Lambda_{n}$ through equation (4), the band-pass filters on all intervals can be expressed as empirical wavelets. The shape of Littlewood-Paley and Meyer wavelet has good compactness in time domain and frequency domain. Its wavelet coefficients can reflect the similarity between the local of the analyzed signal and wavelet basis and highlight the fault information. Based on the idea of constructing Littlewood-Paley and Meyer's wavelets, the scaling function $\widehat{\phi}_{n}(\omega)$ and wavelet function $\widehat{\psi}_{n}(\omega)$ of empirical wavelet are expressed in the frequency domain as follows [26]:

$$
\begin{aligned}
& \widehat{\phi}_{n}(\omega)=\left\{\begin{array}{l}
1, \quad|\omega| \leq(1-\gamma) \omega_{n}, \\
\cos \left[\frac{\pi}{2} \beta\left(\frac{1}{2 \gamma \omega_{n}}\left(|\omega|-(1-\gamma) \omega_{n}\right)\right)\right](1-\gamma), \quad \omega_{n} \leq|\omega| \leq(1+\gamma) \omega_{n}, \\
0, \quad \text { otherwise, }
\end{array}\right. \\
& \widehat{\psi}_{n}(\omega)=\left\{\begin{array}{l}
1, \quad(1+\gamma) \omega_{n} \leq|\omega| \leq(1-\gamma) \omega_{n+1}, \\
\cos \left[\frac{\pi}{2} \beta\left(\frac{1}{2 \gamma \omega_{n+1}}\left(|\omega|-(1-\gamma) \omega_{n+1}\right)\right)\right], \quad(1-\gamma) \omega_{n+1} \leq|\omega| \leq(1+\gamma) \omega_{n+1}, \\
\sin \left[\frac{\pi}{2} \beta\left(\frac{1}{2 \gamma \omega_{n}}\left(|\omega|-(1-\gamma) \omega_{n}\right)\right)\right], \quad(1-\gamma) \omega_{n} \leq|\omega| \leq(1+\gamma) \omega_{n}, \\
0, \quad \text { otherwise, }
\end{array}\right.
\end{aligned}
$$




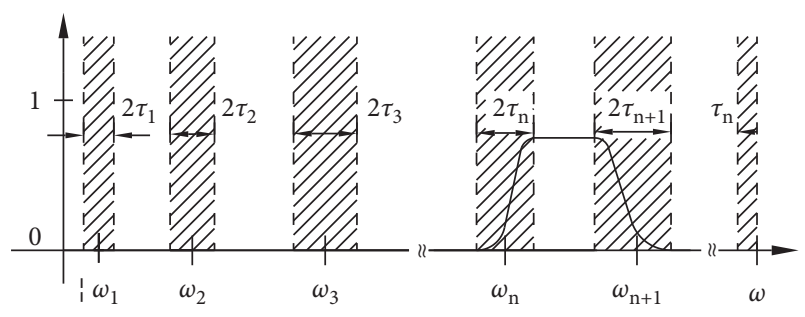

FIgURE 10: Schematic diagram of interval bandwidth.

where $\beta(w)$ is an auxiliary function of a Meyer wavelet between 0 and 1 , which can be expressed by polynomial function as $[28,29]$

$$
\beta(y)=y^{4}\left(35-84 y+70 y^{2}-20 y^{3}\right) .
$$

Define Fourier transform and its inverse transform as $\mathrm{F}$ $[\bullet]$ and $\mathrm{F}^{-1}[\bullet]$, respectively. Assuming that the empirical wavelet transform is $W_{x}^{e}$, the wavelet approximation and detail coefficients, based on the empirical wavelet theory, are calculated through the following inner products:

$$
\begin{aligned}
W_{x}^{e}(0, t) & =\left\langle x, \phi_{1}\right\rangle \\
& =\int x(\tau) \phi_{1}^{*}(\tau-t) \mathrm{d} \tau=F^{-1}\left[X(\omega) \widehat{\phi}_{1}(\omega)\right], \\
W_{x}^{e}(n, t) & =\left\langle x, \psi_{n}\right\rangle \\
& =\int x(\tau) \psi_{n}^{*}(\tau-t) \mathrm{d} \tau=F^{-1}\left[X(\omega) \widehat{\psi}_{n}(\omega)\right],
\end{aligned}
$$

where " $<>$ " represents the inner product; “*” represents conjugate of the mother wavelet function; and $\phi_{1}(t)$ and $\psi_{n}(t)$ are the inverse Fourier transform of the scaling function $\widehat{\phi}_{n}(\omega)$ and wavelet function $\widehat{\psi}_{n}(\omega)$, respectively.

The wavelet packet further decomposes the undecomposed high-frequency contents to improve the time-frequency resolution [30]. Here, we define the subspace $U_{j}^{n}$ as the closure space of the function $u_{n}(t)$, and $U_{j}^{2 n}$ is the closure space of the function $u_{2 n}(t)$; that is, $u_{0}(t)$ represents the empirical scaling function $\widehat{\phi}_{n}(\omega)$, and $u_{1}(t)$ represents the empirical wavelet function $\widehat{\psi}_{n}(\omega)$. $\widehat{\phi}_{n}(\omega)$ and $\widehat{\psi}_{n}(\omega)$ are defined by equations (6a) and (6b), respectively. The decomposition of EWP is obtained in the following form:

$$
\left\{\begin{array}{l}
u_{j, 2 n}(t)=\sqrt{2} \sum_{k \in Z} h(k) u_{n}(2 t-k) \\
u_{j, 2 n+1}(t)=\sqrt{2} \sum_{k \in Z} g(k) u_{n}(2 t-k)
\end{array},\right.
$$

where $g(n)$ is a high-pass wavelet filter related to $\widehat{\psi}_{n}(\omega)$.

The reconstruction function of the wavelet packet is

$$
\begin{aligned}
u_{i, j, 2 n+1}= & \sqrt{2} \sum_{k \in Z} h(k) u_{k, j, n}(2 t-k) \\
& +\sqrt{2} \sum_{k \in Z} g(k) u_{k, j+1, n}(2 t-k),
\end{aligned}
$$

where $u_{i, j, 2 n+1}$ is the $i$-th wavelet packet coefficient of the $n$ th node of the $j$-th level.

Based on EWP, the Fourier function can be converted into empirical mode function, and then the original input signal can be decomposed and filtered to reduce the noise proportion of the original signal. After the original signal is processed by EWP, the input signal set with high signal-tonoise ratio can be obtained. Figure 11 is the comparison diagram of core fault signal envelope spectrum of empirical wavelet packet transform and wavelet packet transform. It can be seen that the amplitude of vibration frequency in the envelope spectrum based on wavelet packet transform is small at $300 \mathrm{~Hz}$, and there is no outstanding performance at 400 and $500 \mathrm{~Hz}$. The vibration frequency changes obviously in the envelope spectrum based on empirical wavelet packet transform, so the fault features have higher resolution based on the EWP transform.

The EWP transform has the advantages of decreasing the number of frequency band dividing points, reducing the amount of calculation, improving the frequency band cracking phenomenon, and more accurate decomposition results. Therefore, the EWP transform can accurately extract various vibration characteristic frequencies of the elevator control transformer, which is beneficial to the resolution of harmonic components and can improve the fault diagnosis efficiency of the elevator control transformer.

3.3. Fault Identification. BP neural network (BPNN) is a multilayer feedforward backpropagation training error algorithm, which is suitable for nonlinear mapping of parameters and has parallelism and strong adaptive learning ability. Its optimization algorithm has been applied to fault diagnosis by many scholars [31-33]. Due to the uncertainty of random search initial weight threshold and parameter structure, the convergence speed of BPNN algorithm is slow and the prediction is unstable. Many scholars use relevant optimization algorithms to improve the efficiency and prediction accuracy of BPNN. Our methodology utilizes mind evolutionary algorithm (MEA) to optimize BP neural network strategy (MEA-BPNN).

MEA is proposed by Sun in 1998 [34], which divides the whole population into subgroups by taking the ideas of "population" and "evolution" in genetic algorithm (GA) as the main body. The convergence and dissimilation are used to replace the crossover and variation in GA. Convergent operation is equivalent to the learning function of the human mind, which occurs within the subgroups. Individuals in each subgroup learn from their winner (the individual with the highest score in the subgroup) in the subgroup and compete with each other until a new winner emerges. The winner's score is the subgroup's score. The sign of the evolutionary maturity of a subgroup is that the winner in the process of convergence no longer changes, which means that the process of the subgroup convergence is over. The dissimilation operation simulates the creative function of the human mind, which is the process of producing a global winner in the entire space. In the process of dissimilation, the temporary subgroup with high scores will continuously replace the temporary subgroup with low scores, while the individuals abandoned by the temporary subgroup with low scores will be released into the whole space for further search, and a new temporary group is formed. 


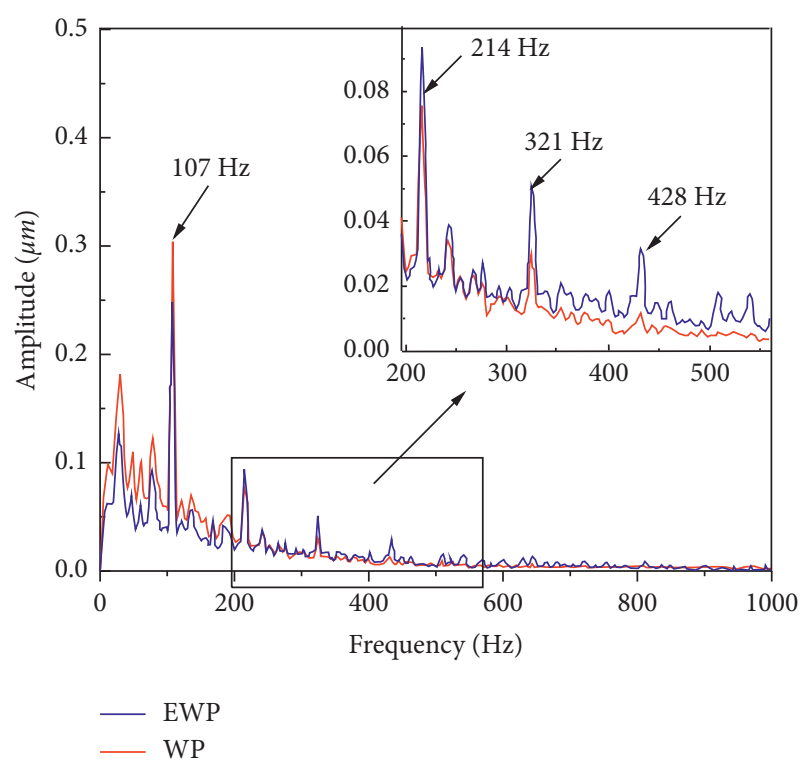

FIGURE 11: Comparison of core fault signal envelope spectrum of EWP and WP.

In general, the idea of MEA can be described as follows.

Step 1: according to the optimized network structure, $S$ individuals are evenly dispersed in the solution space, and the score of each individual is calculated. The score function is determined by the objective evaluation function, which is related to the error of the diagnosis result. Its expression is as follows [35]:

$$
S=\frac{m}{\sum_{i=1}^{m}\left(y_{i}-y_{i}^{*}\right)^{2}},
$$

where $m$ is the number of neural network output nodes, $y_{i}, y_{i}^{*}$ are the true value on test sample set and the diagnostic output value for the $i$-th node of network, respectively.

Step 2: look for Ns individuals with the highest scores (i.e., winners), and publish and sort them on the global bulletin board. Take Ns individuals as the center to form Ns superior subpopulations. Then, $\mathrm{S}$ individuals are randomly scattered in the solution space, and NT winners are selected to form NT temporary subpopulations. Each subpopulation consists of $m$ individuals.

Step 3: perform Similartaxis learning within the winning and temporary groups until the group is mature. Individuals in groups are dispersed among the winners, and they search around the winners and compete with each other to produce new winners. Information about each individual is posted on a local bulletin board, and the score of the group is taken as the score of winner in this group. Step 4: alienation operation is carried out in the global scale. If some temporary group score is higher than that of the winning group, the winning group will be replaced by the temporary group. The individuals in the replaced group are redistributed in solution space in order to get a new temporary group.
Step 5: if this process does not converge, return to Step (3); otherwise, the superior individual is output.

Step 6: the initial values of weight and threshold of BP neural network are given according to the MEA parameter optimization results, and then the MEA-BPNN is trained to diagnose the transformer state.

The optimization architecture of MEA-BPNN is shown in Figure 12.

\section{Experimental Results}

In this paper, the fault diagnosis system of control transformer described above is developed in MATLAB 2020a environment.

4.1. Feature Construction. In the process of transformer operation, the energy and frequency change with the transformer state. In the stable working state, the energy is mainly concentrated on the dominant frequency; in the fault working state, the amplitude of the vibration increases significantly, which means that that energy is transferred. Therefore, it is effective and feasible to judge whether transformer failure occurs in the process of working by means of energy. Before feature extraction, the EWP technique is applied to time series and Daubechies orthogonal wavelet $\mathrm{db} 10$ is used as wavelet basis function. The steps of extracting energy ratio and energy entropy features by EWP can be described as follows:

(1) The vibration signal processed by noise reduction is decomposed by EWP with 3 levels.

(2) Reconstruct the signals of different frequency bands of the third level, and the reconstructed signals are $\left(\mathrm{C}_{30}, \mathrm{C}_{31}, \mathrm{C}_{32}, \ldots, \mathrm{C}_{37}\right)$.

(3) Calculate the energy of each frequency band as $E_{3 j}=\sum_{i=1}^{N}\left|C_{3 j}\right|^{2}, j=0,1,2 \ldots, 7$, where $N$ is the number of signal sampling points.

(4) The total energy can be obtained by $E_{T}=\sum_{j=1\left|E_{3 j}\right|}^{2}$.

(5) According to the Shannon entropy theory, calculate the wavelet entropy [36] of the vibration signal by $E E=-\sum_{j} T_{3 j} \lg T_{3 j}$, where $T_{3 j}=E_{3 j} / E_{T}$ describes percentage of energy of each frequency band for the whole signal. Finally, the feature vector $\mathrm{e}=\left[T_{31}, T_{32}\right.$, $\left.T_{33}, T_{34}, T_{35}, T_{36}, T_{37}, T_{38}, E E\right]$ is obtained as the input of the MEA-BPNN neural network.

To analyze EWP performance, the EWP coefficients of levels 1, 2, and 3 are obtained from experimental data. Figure 13 shows the level 3 energy ratio and energy entropy of EWP in four states.

From Figure 13, it can be found that, for stable running condition, most of the energy is concentrated in the first, second, and third wavelet packets. For the high-order harmonic running condition the energy is concentrated mainly in the fourth, fifth, and sixth wavelet packets, and most of the energy is concentrated in the sixth, seventh, and eighth wavelet packets for fault states. At the same time, it is obviously found that the energy entropy of empirical wavelet 


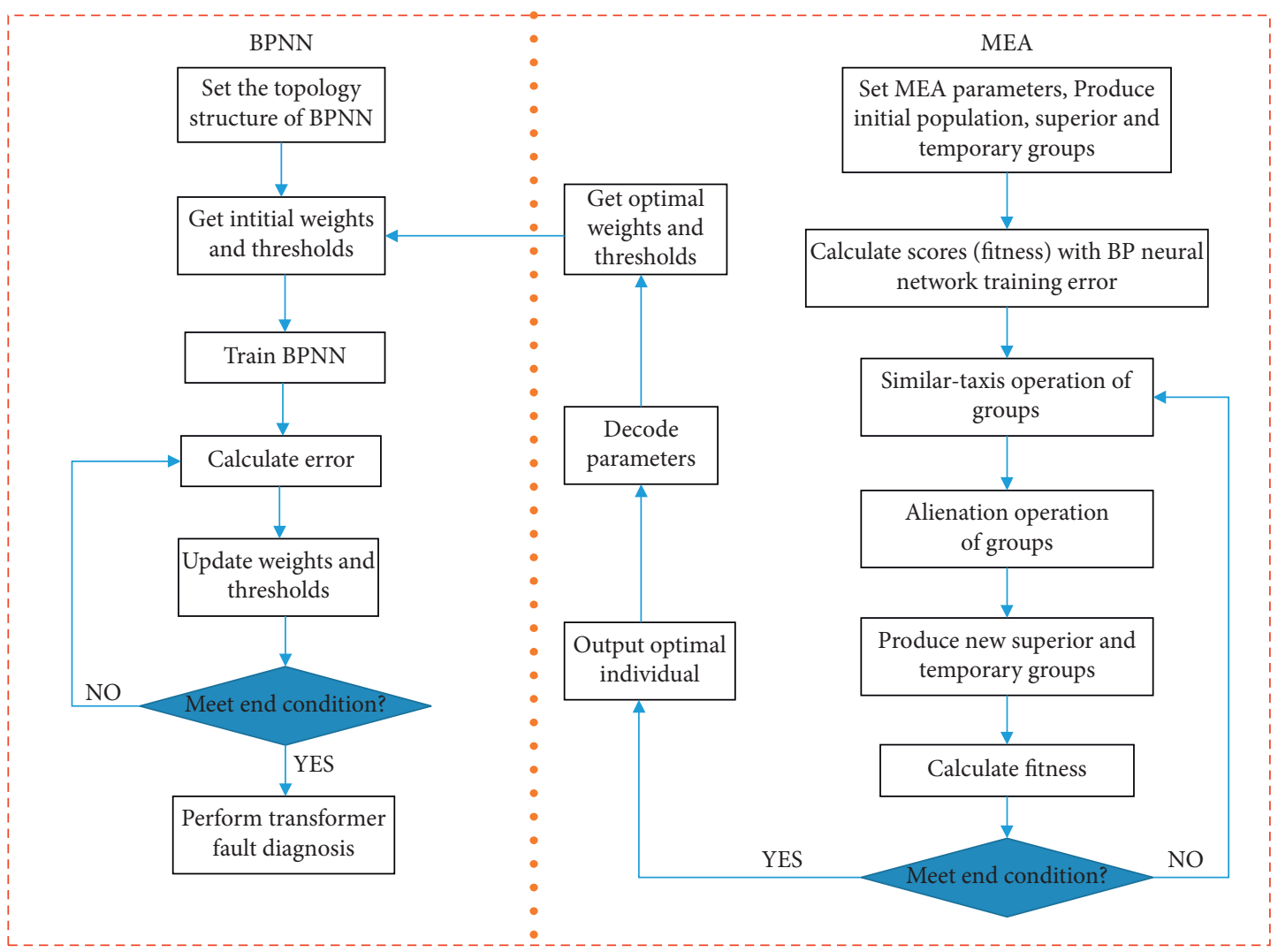

FIGURE 12: Global flowchart of MEA-BPNN method.

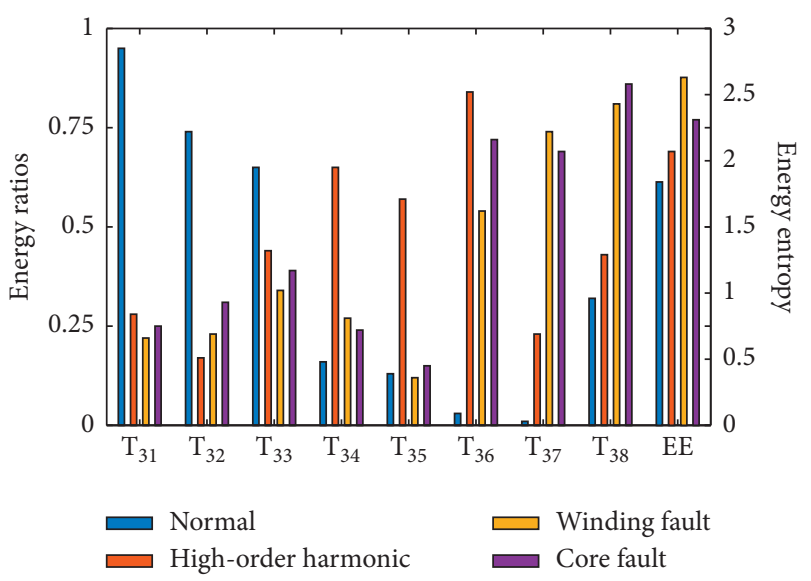

FIgURE 13: Energy ratios of empirical wavelet packets for four states.

packet of vibration signal can reflect the changes of different states during transformer operation, which can be used for transformer state diagnosis.

\subsection{Experimental Analysis and Discussion}

4.2.1. Parameter Performance Analysis of MEA-BPNN. In MEA, the number of individuals in the subgroup $(m)$ is the number of features included in each similartaxis process. When the value is relatively small, the features with low similarity can be distinguished clearly; however, it has a large number of evolution times or is difficult to converge. If the value is relatively large, because its tolerance for features with low similarity is in the form of percentage, it will tolerate too many features with low similarity, resulting in matching errors; however, it has a smaller number of evolution times. The experimental results of changing $m$ value are shown in Table 2.

All feature points are treated with similartaxis and dissimilation operations once, which is regarded as an evolution. In the vibration signal of control transformer, the signal requiring the least number of evolution times has the minimum number of evolution times, and the signal requiring the largest number of evolution times has the maximum number of evolution times, and the upper limit of evolution times is 50 . The correct convergence rate is that the convergence is completed within the limited evolution times, and the number of corresponding relations of feature points is greater than $75 \%$ of the number of all feature points. It can be seen from the results that, for the vibration signal of elevator control transformer, when $m$ is selected as 5,6 , and 7 , the number of evolutions is small and the correct convergence rate is large, so the system has better performance. Here 7 individuals are selected in the subgroup.

For the number of initial subpopulation $(w)$, it represents the number of initial benchmark feature points. When its value is relatively large, there are more initial populations and more similartaxis processes. When its value is relatively 
TABLE 2: Influence of the number of individuals in a subgroup on system performance.

\begin{tabular}{lcccc}
\hline $\begin{array}{l}\text { Number } \\
\text { of } m\end{array}$ & $\begin{array}{c}\text { Minimum } \\
\text { evolution } \\
\text { times }\end{array}$ & $\begin{array}{c}\text { Maximum } \\
\text { evolution } \\
\text { times }\end{array}$ & $\begin{array}{c}\text { Average } \\
\text { evolution } \\
\text { times }\end{array}$ & $\begin{array}{c}\text { Correct } \\
\text { convergence } \\
\text { rate (\%) }\end{array}$ \\
\hline 3 & 23 & 49 & 40 & 22.48 \\
4 & 22 & 45 & 39 & 61.91 \\
5 & 21 & 40 & 34 & 79.18 \\
6 & 20 & 38 & 32 & 81.67 \\
7 & 18 & 36 & 32 & 82.46 \\
8 & 19 & 33 & 30 & 68.73 \\
\hline
\end{tabular}

small, there are more dissimilation processes, and the unilateral increase of similartaxis process or dissimilation process will reverse after evolution to a certain period of time. In other words, there are many similartaxis processes at the beginning; however, the number of temporary populations decreases sharply when a large number of feature points are included in the similartaxis process, the number of temporary populations decreases sharply, and then a large number of dissimilation processes begin to appear. After a large number of dissimilation processes, a considerable number of temporary groups will lead to a large number of similartaxis processes. Although this phenomenon will become weaker and weaker and eventually converge, it obviously leads to the increase of operation time. The experimental results of changing $w$ value are shown in Table 3.

With the change of the number of selected initial populations, it can be seen from Table 3 that, after training for the same number of times, when 20-25 initial populations of mind evolutionary are selected, the performance of the system is better. Here, 25 initial populations are selected.

Generally, the number of hidden layer nodes of BP neural network is determined according to the empirical formula, as follows:

$$
H=\sqrt{P+Q}+A,
$$

where $H$ is the number of hidden layer nodes, $P$ is the number of nodes in the input layer, $Q$ is the number of output nodes, and $A$ is a constant between 1 and 10 .

According to equation (12), constant training experiments with $A$ between 1 and 10 are selected, respectively. When the number of hidden layer nodes is 7 , the prediction of BP neural network model is more accurate and the learning efficiency is higher. So the MEA-BPNN model adopts 9-7-3 three-layer BP neural network structure. The other parameters of MEA-BP are shown in Table 4. The input layer inputs the nine feature parameters of energy and energy entropy of EWP, and the hidden layer node is the empirical wavelet basis function; the output layer outputs the four diagnosis results: normal state, high-order harmonic state, winding, and core fault states.

4.2.2. Correlation Analysis of Diagnostic Results. During comparative experimental process of BPNN and MEA-
TABle 3: Training parameter corresponding to different initial population.

\begin{tabular}{lcccc}
\hline $\begin{array}{l}\text { Number of initial } \\
\text { populations }\end{array}$ & $\begin{array}{c}\text { Training } \\
\text { step }\end{array}$ & $\begin{array}{c}\text { Training } \\
\text { time }\end{array}$ & $\begin{array}{c}\text { Average } \\
\text { evolution } \\
\text { times }\end{array}$ & $\begin{array}{c}\text { Training } \\
\text { error }\end{array}$ \\
\hline 10 & 24 & 196.6149 & 45 & 0.01729371 \\
15 & 32 & 260.3514 & 40 & 0.01305725 \\
20 & 46 & 323.5294 & 36 & 0.00972516 \\
25 & 86 & 340.1963 & 31 & 0.00865904 \\
30 & 114 & 549.2634 & 34 & 0.01863492 \\
35 & 157 & 812.5183 & 39 & 0.09075341 \\
\hline
\end{tabular}

BPNN, we randomly divided the samples into three groups: the training samples, the test samples, and the validation samples. The performance of MEA-BPNN model is verified by using the determination coefficient $(R)$ index, which is defined as [35]

$$
R=1-\sqrt{\frac{\sum_{i=1}^{n}\left(y_{i}-y_{i}^{*}\right)^{2}}{\sum_{i=1}^{n}\left(y_{i}-\bar{y}\right)^{2}}},
$$

where $n$ is the number of samples, $y_{i}, y_{i}^{*}$ are the true value on test sample set and the diagnostic output value, respectively, and $\bar{y}$ is the average of the true values on the test sample set.

The determination coefficient $(R)$ represents the quality of a fitting through changes in the data. The value of $R$ is close to 1 , indicating that the model has the higher reliability; the value of $R$ is far less than 0 , indicating that the reliability of the model is very poor. Figure 14 presents the determination coefficients between the diagnostic output and the experimental measured value.

Comparatively speaking, the BPNN model has a poor effect in training, verification, and testing stages, which indicates that the generalization ability of BPNN needs to be improved. However, the diagnostic performance and results of MEA-BPNN are relatively balanced in three stages of training, verification, and testing, and its effect is better than that of BPNN model, which means that MEA improves BPNN generalization ability. Similartaxis and alienation learning in MEA can not only improve the global search ability, but also improve the generalization ability of MEABPNN.

Using the measured experimental data, the established MEA-BPNN transformer fault diagnosis model is trained and tested. To compare the performance of the MEA-BPNN model proposed in this article, the root mean square error (RMSE) is used to evaluate the diagnosis results. The calculation formula is as follows:

$$
\text { RMSE }=\sqrt{\frac{1}{n} \sum_{i=1}^{n}\left(y_{i}-y_{i}^{*}\right)^{2}},
$$

where $y_{i}$ is the actual value of the test data and $y_{i}^{*}$ is the diagnosis result.

The experimental result is given in Table 5. In the MEABPNN training, the average number of iterations and the average training time are $44.4 \mathrm{~s}$ and $4.39 \mathrm{~s}$, respectively, 
TAble 4: Parameters of MEA-BP model.

\begin{tabular}{lccc}
\hline Parameters & Initial value & Parameters & Initial value \\
\hline Learning rate & 0.04 & Number of superior subgroups & 5 \\
Convergence error & 0.001 & Number of temporary subgroups & 5 \\
Score of superior subgroup & 2 & Maximal iterative number & 12 \\
Score of temporary subgroup & 4 & Population size & 150 \\
\hline
\end{tabular}
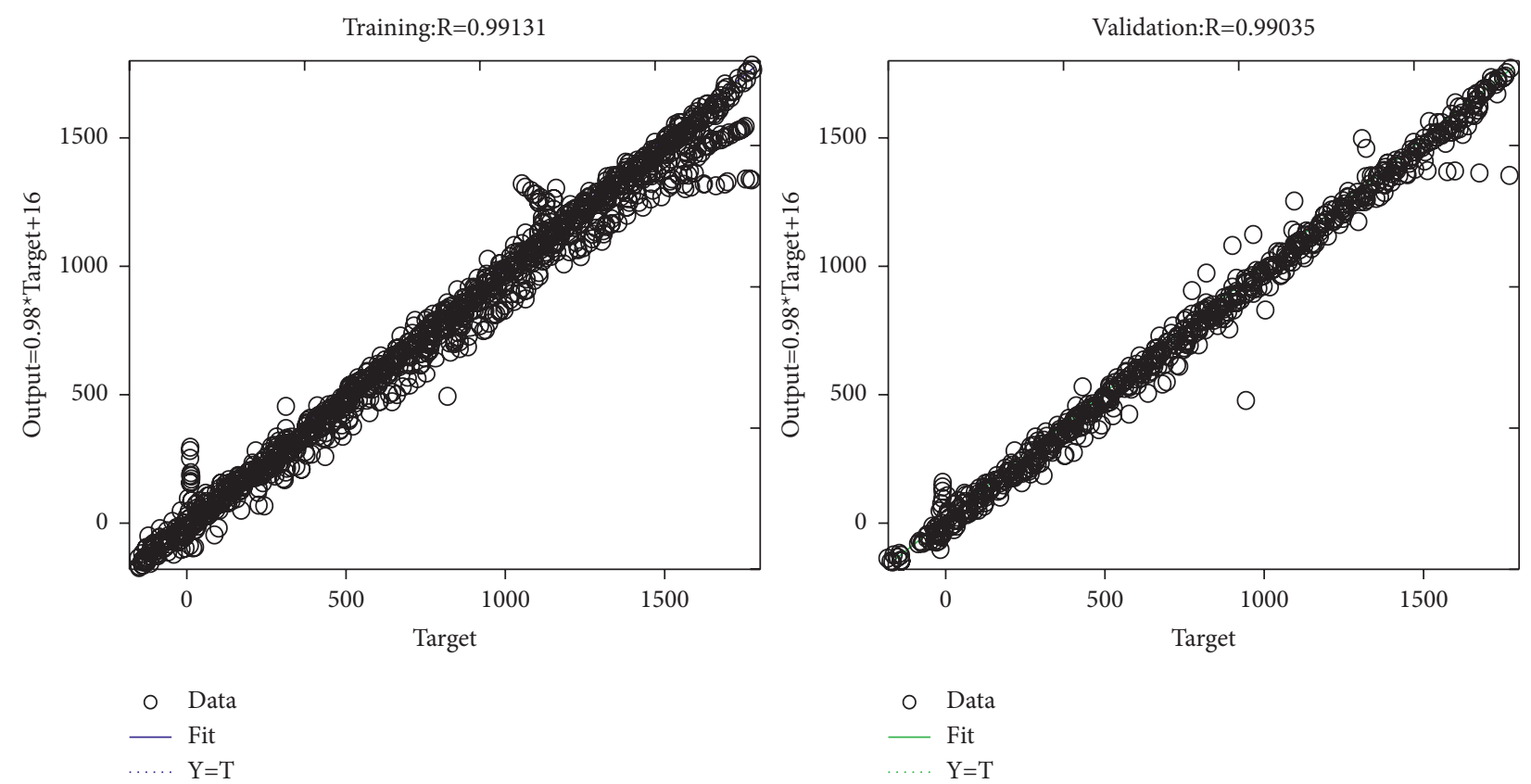

Test: $\mathrm{R}=0.99139$
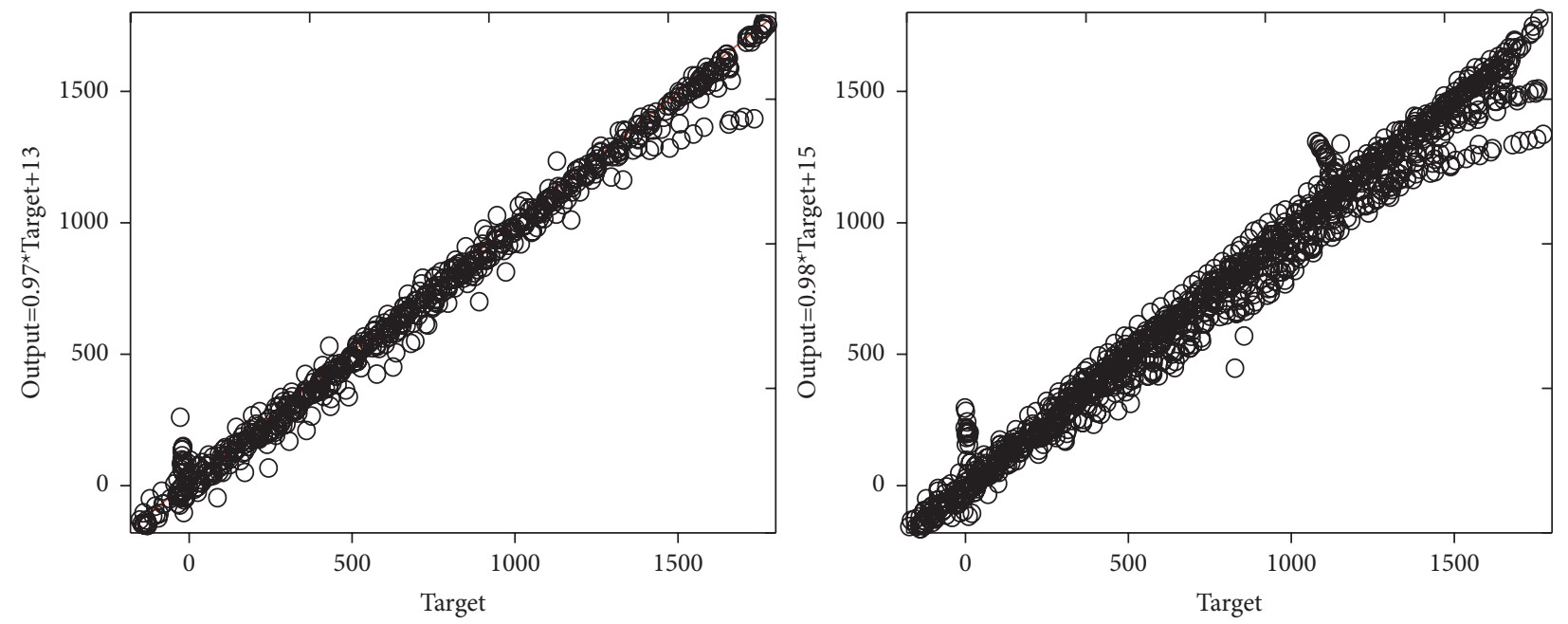

O Data

- Fit

○ Data

- Fit

. $\mathrm{Y}=\mathrm{T}$

(a)

FIgURE 14: Continued. 

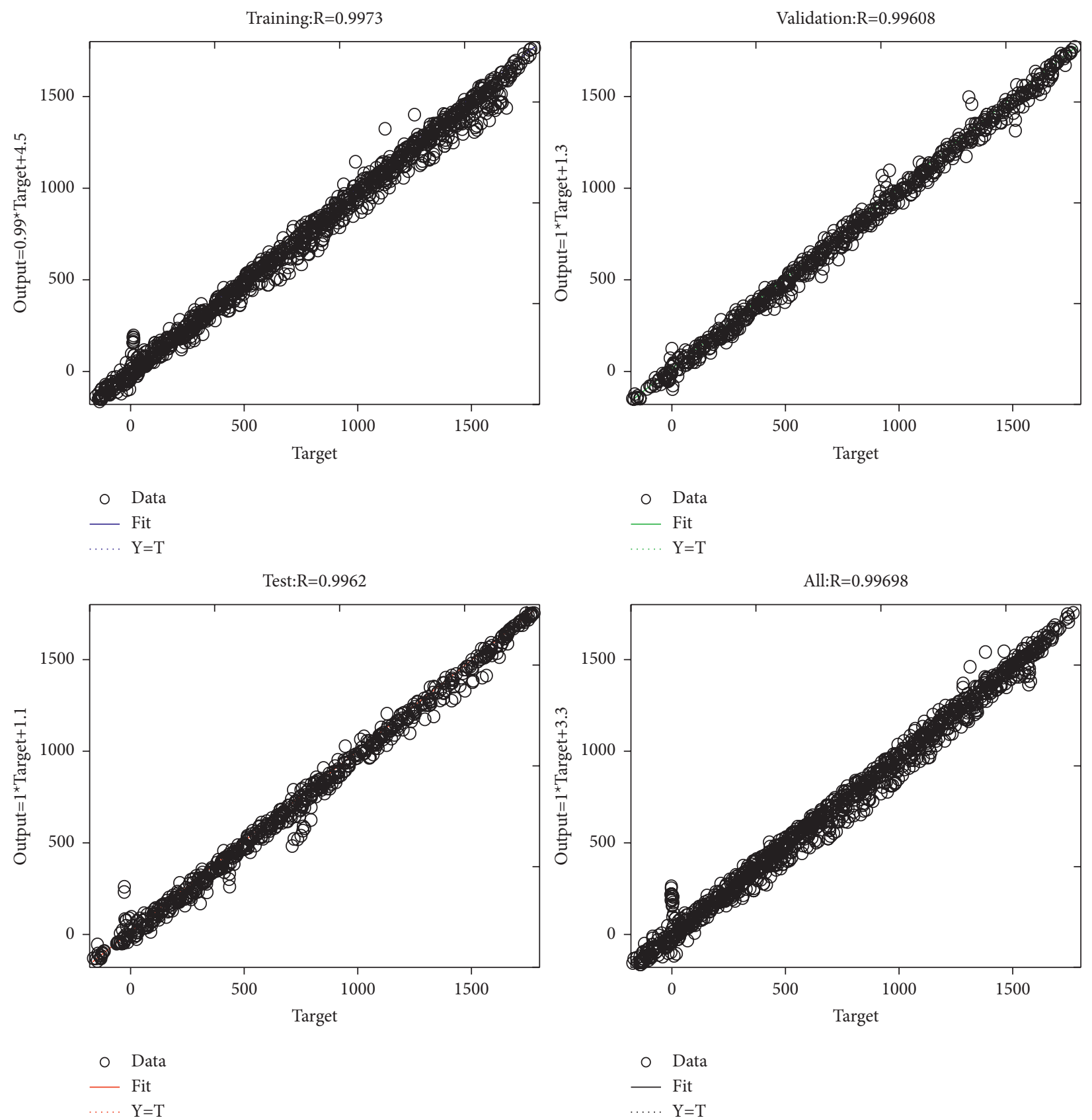

(b)

FIGURE 14: Scatter plot of models and determination coefficient. (a) BPNN; (b) MEA-BPNN.

which are better than BPNN and GA-BPNN. The average root mean square error of MEA-BPNN is about $0.007 \mu \mathrm{m}$ which can meet the accuracy requirements of fault diagnosis and quality evaluation, and that of GA-BPNN is about $0.05 \mu \mathrm{m}$, and that of BPNN is about $0.08 \mu \mathrm{m}$, which shows that the MEA-BPNN model has high diagnosis accuracy. The MEA algorithm based on the GA adopts the similartaxis and alienation operations, which can ensure high-efficiency global search and significantly improve the disadvantage of poor local search ability of genetic algorithm. Moreover, the number of parameters to be adjusted in thinking evolution is less, which is simpler and faster than genetic algorithm. The prediction accuracy and training speed of the optimized neural network are improved to a certain extent.

4.2.3. Diagnosis Results and Comparative Analysis. To compare the performance of the WP-BPNN model, the WPMEA-BP model, the EWP-BPNN model, and the EWPMEA-BPNN model, the experimental cases based on the original transformer vibration time series are proposed in this section. Figure 15 shows the diagnosis result of the highorder harmonic running condition. The BP neural network model optimized by MEA not only realizes better 
TABLE 5: Data for model diagnostic results.

\begin{tabular}{|c|c|c|c|c|c|c|c|c|c|}
\hline \multirow[b]{2}{*}{ No. } & \multicolumn{3}{|c|}{ BPNN } & \multicolumn{3}{|c|}{ GA-BPNN } & \multicolumn{3}{|c|}{ MEA-BPNN } \\
\hline & $\begin{array}{c}\text { RMSE } \\
(\mu \mathrm{m})\end{array}$ & $\begin{array}{c}\text { Number of } \\
\text { iterations }\end{array}$ & $\begin{array}{l}\text { Training } \\
\text { time (s) }\end{array}$ & $\begin{array}{c}\text { RMSE } \\
(\mu \mathrm{m})\end{array}$ & $\begin{array}{c}\text { Number of } \\
\text { iterations }\end{array}$ & $\begin{array}{l}\text { Training } \\
\text { time (s) }\end{array}$ & $\begin{array}{c}\text { RMSE } \\
(\mu \mathrm{m})\end{array}$ & Number of iterations & $\begin{array}{l}\text { Training } \\
\text { time (s) }\end{array}$ \\
\hline 1 & 0.04172 & 96 & 7.3 & 0.02319 & 87 & 5.6 & 0.00163 & 56 & 4.7 \\
\hline 2 & 0.01914 & 72 & 8.5 & 0.04127 & 65 & 4.9 & 0.00844 & 52 & 4.5 \\
\hline 3 & 0.05737 & 56 & 7.8 & 0.02896 & 54 & 5.2 & 0.00493 & 49 & 5.1 \\
\hline 4 & 0.05132 & 79 & 8.9 & 0.03655 & 80 & 7.1 & 0.00772 & 59 & 5.7 \\
\hline 5 & 0.08451 & 82 & 7.4 & 0.06261 & 91 & 4.6 & 0.02164 & 35 & 3.4 \\
\hline 6 & 0.03727 & 83 & 7.9 & 0.08175 & 70 & 6.3 & 0.01009 & 41 & 3.7 \\
\hline 7 & 0.03236 & 79 & 9.5 & 0.05286 & 66 & 4.8 & 0.00103 & 49 & 4.1 \\
\hline 8 & 0.07153 & 113 & 8.7 & 0.06103 & 59 & 5.4 & 0.00115 & 39 & 3.9 \\
\hline 9 & 0.30247 & 87 & 8.4 & 0.04905 & 63 & 5.7 & 0.00121 & 34 & 4.2 \\
\hline 10 & 0.08463 & 91 & 9.3 & 0.03617 & 74 & 4.7 & 0.00936 & 30 & 4.6 \\
\hline Mean & 0.07823 & 83.8 & 8.37 & 0.04734 & 70.9 & 5.43 & 0.00672 & 44.4 & 4.39 \\
\hline
\end{tabular}

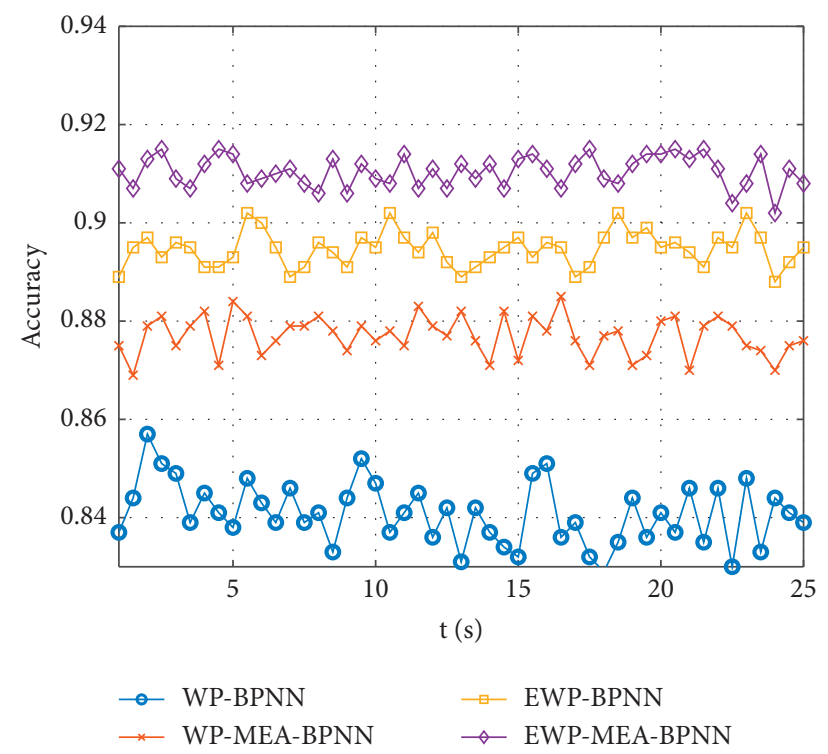

FIGURE 15: The diagnosis result for the high-order harmonic running condition.

optimization of the initial weight and threshold of the input layer, but also shows strong global search ability, which significantly improves the diagnostic accuracy of the model. The accuracy of diagnosis depends not only on the classification algorithm, but also on the extracted features. EWP is used to process the original input signal, reduce the noise proportion in the signal, and obtain the input signal set with high signal-to-noise ratio. In MEA-BPNN model, EWP is better than WP in extracting wavelet packet coefficient energy and energy entropy as features. Combined with Table 6 , it is found that, in MEA-BPNN, the empirical wavelet packet feature extraction can obtain higher average accuracy in transformer fault diagnosis compared with wavelet packet feature extraction; the diagnostic average accuracy of the four states was improved by $3.3 \%, 3.4 \%$, $3.6 \%$, and $3.3 \%$, respectively.

In order to verify the performance of EWP-MEA-BPNN, it operates independently for 30 times under normal, harmonic, and fault conditions. The average, minimum, and maximum values of accuracy are shown in Table 6 .
It can be seen from Table 6 that WP-BPNN model has the lowest diagnostic accuracy of transformer state. The average diagnostic accuracy of this method for normal, harmonic, and fault states of transformer is $84.3 \%, 84.1 \%$, $83.7 \%$, and $84.1 \%$, respectively. For the above four state types, the average accuracy of EWP-BPNN is $84.9 \%$, $89.2 \%, 89.1 \%$, and $88.9 \%$, and the WP-MEA-BPNN and EWP-MEA-BPNN are $87.7 \%, 87.4 \%, 87.3 \%, 87.6 \%$ and $91 \%$, 90.4\%, 90.3\%, 90.5\%, respectively. However, for harmonics, due to frequency aliasing and irrelevant interference frequency in the signal decomposition results, the traditional wavelet packet cannot be separated accurately. The empirical wavelet packet method can accurately analyze the harmonic components of the experimental signal and improve the diagnostic accuracy. At the same time, EWP-MEA-BPNN model is applied to four states, and the maximum, minimum, and average accuracy are the maximum. Therefore, the model optimized by mind evolutionary algorithm improves the diagnosis accuracy. 
TABLe 6: Diagnostic accuracy of the models for different operating states after 30 independent runs.

\begin{tabular}{lccccccccccccc}
\hline \multirow{2}{*}{ Algorithm } & \multicolumn{3}{c}{ Normal } & \multicolumn{4}{c}{ High-order harmonic } & \multicolumn{3}{c}{ Winding fault } & \multicolumn{3}{c}{ Core fault } \\
& Mean & Min & Max & Mean & Min & Max & Mean & Min & Max & Mean & Min & Max \\
& 0.843 & 0.833 & 0.875 & 0.841 & 0.832 & 0.877 & 0.837 & 0.830 & 0.874 & 0.841 & 0.829 & 0.877 \\
WP-BPNN & 0.877 & 0.869 & 0.884 & 0.874 & 0.862 & 0.883 & 0.871 & 0.863 & 0.884 & 0.876 & 0.835 & 0.885 \\
WP-MEA-BPNN & 0.849 & 0.837 & 0.881 & 0.892 & 0.887 & 0.902 & 0.891 & 0.886 & 0.900 & 0.889 & 0.842 & 0.893 \\
EWP-BPNN & 0.910 & 0.905 & 0.915 & 0.908 & 0.904 & 0.913 & 0.907 & 0.903 & 0.912 & 0.905 & 0.899 & 0.917 \\
EWP-MEA-BPNN & 0.910 &
\end{tabular}

\section{Conclusions}

In order to realize fault detection of elevator transformer vibration, a fault diagnosis analysis method combining empirical wavelet packet transform and MEA-BPNN was proposed based on transformer surface vibration signals in this paper. The transformer fault diagnosis model experiments are carried out, and the results are in good agreement with the simulation results. The following main conclusions were obtained:

(1) The method of empirical wavelet packet transform is used to process the vibration signal of elevator control transformer, which improves the problem of insufficient adaptability of wavelet packet transform in frequency band division. The features of energy and energy entropy of empirical wavelet packet coefficients are extracted to improve the accuracy of transformer state diagnosis.

(2) MEA is used to optimize BP neural network algorithm, which can improve the local search ability of the algorithm and ensure higher global search efficiency. The network diagnosis accuracy and training speed are better than BP neural network.

(3) The diagnostic model about MEA-BPNN combined with energy and energy entropy of EWP is applied to the experimental operation of the elevator transformer, which has higher efficiency and diagnostic accuracy, compared with the wavelet packet technology and BP neural network model.

(4) In the process of model establishment and experiment, it is found that there are differences in diagnostic accuracy of different operating states, which is mainly due to the strong dependence of neural network on sample data. Therefore, future work should include collecting more actual operating conditions of elevator control transformer. Furthermore, studying better feature extraction methods and fault diagnosis algorithms is also interesting topics which are our ongoing works.

\section{Data Availability}

The data used to support the findings of this study are available from the corresponding author upon request.

\section{Conflicts of Interest}

The authors declare that there are no conflicts of interest in the publication of this paper.

\section{Acknowledgments}

This work was supported by the National Natural Science Foundation of China (51774193 and 52174145), Natural Science Foundation of Shan-dong Provincial China (ZR2020MF101), University Planning Projects of Science and Technology of Shandong Province (J18KB011 and J18KA319), and SDUST Research Fund (2018TDJH101).

\section{References}

[1] X. W. Zhang and H. S. Li, "Research on transformer fault diagnosis method and calculation model by using fuzzy data fusion in multi sensor detection system," Optik, vol. 176, pp. 716-723, 2019.

[2] A. Zou, D. Rui, Q. Mei, and Z. Lang, "Fault diagnosis of a transformer based on polynomial neural networks," Cluster Computing, vol. 22, pp. S9941-S9949, 2019.

[3] A. Li, X. Yang, Z. Xie, and C. Yang, "An optimized GRNNenabled approach for power transformer fault diagnosis," IEEJ Transactions on Electrical and Electronic Engineering, vol. 14, no. 8, pp. 1181-1188, 2019.

[4] X. Yang, W. Chen, A. Li, C. Yang, Z. Xie, and H. Dong, "BAPNN-based methods for power transformer fault diagnosis," Advanced Engineering Informatics, vol. 39, pp. 178-185, 2019.

[5] X. Yang, W. Chen, A. Li, and C. Yang, "A Hybrid machinelearning method for oil-immersed power transformer fault diagnosis," IEEJ Transactions on Electrical and Electronic Engineering, vol. 15, no. 4, pp. 501-507, 2020.

[6] B. Zhao, M. Yang, H. R. Diao, B. An, Y. C. Zhao, and Y. M. Zhang, "A novel approach to transformer fault diagnosis using IDM and naive credal classifier," International Journal of Electrical Power \& Energy Systems, vol. 105, pp. 846-855, 2019.

[7] A. Hoballah, D. E. A. Mansour, and I. B. M. Taha, "Hybrid grey wolf optimizer for transformer fault diagnosis using dissolved gases considering uncertainty in measurements," IEEE Access, vol. 8, pp. 139176-139187, 2020.

[8] S. Li, G. Wu, B. Gao, C. Hao, D. Xin, and X. Yin, "Interpretation of DGA for transformer fault diagnosis with complementary SaE-ELM and arctangent transform," IEEE Transactions on Dielectrics and Electrical Insulation, vol. 23, no. 1, pp. 586-595, 2016.

[9] K. Hong, H. Huang, and J. Zhou, "Winding condition assessment of power transformers based on vibration correlation," IEEE Transactions on Power Delivery, vol. 30, no. 4, pp. 1735-1742, 2015.

[10] Y. Wang and P. Jie, "Comparison of mechanically and ElectricallyExcited vibration frequency responses of a small DistributionTransformer," IEEE Transactions on Power Delivery, vol. 32, no. 3, pp. 1173-1180, 2015.

[11] C. Bartoletti, M. Desiderio, D. Di Carlo et al., "Vibro-acoustic techniques to diagnose power transformers," IEEE Transactions on Power Delivery, vol. 19, no. 1, pp. 221-229, 2004. 
[12] H. Zhou, K. Hong, H. Huang, and J. Zhou, "Transformer winding fault detection by vibration analysis methods," Applied Acoustics, vol. 114, pp. 136-146, 2016.

[13] X. Liu, Y. Yang, Y. Huang, and A. Jadoon, "Vibration characteristic investigation on distribution transformer influenced by DC magnetic bias based on motion transmission model," International Journal of Electrical Power \& Energy Systems, vol. 98, pp. 389-398, 2018.

[14] F. Zhang, S. Ji, Y. Shi, C. Zhan, and L. Zhu, "Investigation on vibration source and transmission characteristics in power transformers," Applied Acoustics, vol. 151, pp. 99-112, 2019.

[15] C. Cao, B. Xu, and X. Li, "Monitoring method on loosened state and deformational fault of transformer winding based on vibration and reactance information," IEEE Access, vol. 8, pp. 215479-215492, 2020.

[16] Y. Konishi, M. Kato, M. Hasegawa, H. Aoyama, and S. Matsumoto, "Tank vibration analysis of an extra-highvoltage transformer," IEEJ Transactions on Electrical and Electronic Engineering, vol. 15, pp. 781-788, 2020.

[17] K. Hong, J. Pan, and M. Jin, “Transformer condition monitoring based on load-varied vibration response and GRU neural networks," IEEE Access, vol. 8, pp. 178685-178694, 2020.

[18] A. Zollanvari, K. Kunanbayev, S. Akhavan Bitaghsir, and M. Bagheri, "transformer fault prognosis using deep recurrent neural network over vibration signals," IEEE Transactions on Instrumentation and Measurement, vol. 70, pp. 1-11, 2021.

[19] J. Wu, Y. Fan, J. Wang et al., "Transportation monitoring of geo-location, speed, vibration and shock acceleration for $110 \mathrm{kV}$ vehicular mobile transformers," IEEE Transactions on Instrumentation and Measurement, vol. 70, Article ID 5500110, 2021.

[20] C. Zhang, Y. He, B. Du, L. Yuan, B. Li, and S. Jiang, "Transformer fault diagnosis method using IoT based monitoring system and ensemble machine learning," Future Generation Computer Systems, vol. 108, pp. 533-545, 2020.

[21] K. Hong, M. Jin, and H. Huang, "Transformer winding fault diagnosis using vibration image and deep learning," IEEE Transactions on Power Delivery, vol. 36, no. 2, pp. 676-685, 2021.

[22] R. Xiao, Z. Zhang, Y. Wu, P. Jiang, and J. Deng, "Multi-scale information fusion model for feature extraction of converter transformer vibration signal," Measurement: Journal of the International Measurement Confederation, vol. 180, pp. 1-12, Article ID 109555, 2021.

[23] M. Bagheri, M. S. Naderi, and T. Blackburn, "Advanced transformer winding deformation diagnosis: moving from off-line to on-line," IEEE Transactions on Dielectrics and Electrical Insulation, vol. 19, no. 6, pp. 1860-1870, 2012.

[24] Y. Chen, Y. Cheng, and H. Liu, "Application of improved wavelet adaptive threshold de-noising algorithm in FBG demodulation," Optik, vol. 132, pp. 243-248, 2017.

[25] C. Barile, C. Casavola, G. Pappalettera, C. Pappalettere, and P. K. Vimalathithan, "Detection of damage in CFRP by wavelet packet transform and empirical mode decomposition: an hybrid approach," Applied Composite Materials, vol. 27, no. 5, pp. 641-655, 2020.

[26] J. Gilles, "Empirical wavelet transform," IEEE Transactions on Signal Processing, vol. 61, no. 16, pp. 3999-4010, 2013.

[27] Z. Mo, H. Zhang, J. Wang, J. Wang, H. Fu, and Q. Miao, "Adaptive Meyer wavelet filters for machinery fault diagnosis based on harmonic infinite-taxicab norm and grasshopper optimization algorithm," Proceedings of the Institution of Mechanical Engineers - Part C: Journal of Mechanical Engineering Science, vol. 235, no. 19, pp. 1-17, 2020.

[28] M. Wang and X. Sheng, "Combining empirical wavelet transform and transfer matrix or modal superposition to reconstruct responses of structures subject to typical excitations," Mechanical Systems and Signal Processing, vol. 163, pp. 1-28, Article ID 108162, 2022.

[29] A. Karimpour and S. Rahmatalla, "Extended empirical wavelet transformation: application to structural updating," Journal of Sound and Vibration, vol. 500, pp. 1-19, Article ID 116026, 2021.

[30] L. Q. Zuo, H. M. Sun, Q. C. Mao, X. Y. Liu, and R. S. Jia, "Noise suppression method of microseismic signal based on complementary ensemble empirical mode decomposition and wavelet packet threshold," IEEE Access, vol. 7, pp. 176504176513, 2019.

[31] I. Djelloul, Z. Sari, and K. Latreche, "Uncertain fault diagnosis problem using neuro-fuzzy approach and probabilistic model for manufacturing systems," Applied Intelligence, vol. 48, no. 9, pp. 3143-3160, 2018.

[32] T. Guo, S. Chang, Z. Chen, H. Huang, and J. Xu, "Fault monitoring and diagnosis of actuators in electromagnetic valve-train based on neural networks optimization algorithm," IEEE Access, vol. 7, pp. 110616-110627, 2019.

[33] B. Cai, X. Sun, J. Wang et al., "Fault detection and diagnostic method of diesel engine by combining rule-based algorithm and BNs/BPNNs," Journal of Manufacturing Systems, vol. 57, no. 7, pp. 148-157, 2020.

[34] C. Sun, "Mind-evolution based machine learning: framework and the implementation of optimization," in Proceedings of the IEEE International Conference on Intelligent Engineering Systems, pp. 355-359, Vienna, Austria, 1998.

[35] Z. Wang, G. Ma, D. Gong, J. Sun, and D. Zhang, “Application of mind evolutionary algorithm and artificial neural networks for prediction of profile and flatness in hot strip rolling process," Neural Processing Letters, vol. 50, no. 3, pp. 2455-2479, 2019.

[36] Z. Gao, S. Li, Q. Cai et al., "Relative wavelet entropy complex network for improving EEG-based fatigue driving classification," IEEE Transactions on Instrumentation and Measurement, vol. 68, no. 7, pp. 2491-2497, 2018. 